\title{
The van der Waals Limit for Classical Systems. I. A Variational Principle
}

\author{
D. J. Gates and O. Penrose \\ Mathematics Department, Imperial College, London, S.W.7
}

Received August 12, 1969

\begin{abstract}
We consider the thermodynamic pressure $p(\mu, \gamma)$ of a classical system of particles with the two-body interaction potential $q(\mathbf{r})+\gamma^{v} K(\gamma \mathbf{r})$, where $v$ is the number of space dimensions, $\gamma$ is a positive parameter, and $\mu$ is the chemical potential. The temperature is not shown in the notation. We prove rigorously, for hard-core potentials $q(\mathbf{r})$ and for a very general class of functions $K(\mathbf{s})$, that the limit $\gamma \rightarrow 0$ of the pressure $p(\mu, \gamma)$ exists and is given by

$$
\sup _{n \in \mathscr{R}} \lim _{|D| \rightarrow \infty} \frac{1}{|D|}\left[\int_{D} d \mathbf{y}\left\{\mu n(\mathbf{y})-a^{0}[n(\mathbf{y})]\right\}-\frac{1}{2} \int_{D} d \mathbf{y} \int_{D} d \mathbf{y}^{\prime} n(\mathbf{y}) n\left(\mathbf{y}^{\prime}\right) K\left(\mathbf{y}-\mathbf{y}^{\prime}\right)\right]
$$

where the limit and the supremum can be interchanged. Here $\mathscr{R}$ is a certain class of nonnegative, Riemann integrable functions, $D$ is a cube of volume $|D|$, and $a^{0}(\varrho)$ is the free energy density of a system with $K=0$ and density $\varrho$. A similar result is proved for the free energy.
\end{abstract}

\section{Introduction}

Many authors have considered the equilibrium statistical mechanics of a system of identical particles which have a two-body interaction potential of the form

$$
v(\mathbf{r}, \gamma)=q(\mathbf{r})+\gamma^{v} K(\gamma \mathbf{r})
$$

where $\mathbf{r}$ is the vector distance between a pair of particles, $\gamma$ is a positive parameter and $v$ is the number of dimensions. The function $q(\mathbf{r})$ is called the short range or reference potential and the term $\gamma^{v} K(\gamma \mathbf{r})$ is called the long range or Kac potential, whose range is proportional to $\gamma^{-1}$. Some of these authors [1-4] have considered the limiting values of the thermodynamic functions and correlation functions in the limit $\gamma \rightarrow 0$; others $[3,5-7]$ have derived expansions of these functions in powers of $\gamma$. We shall be dealing with the former problem. In particular, we shall generalize the results of Lebowitz and Penrose [4] (henceforth referred to as LP) to a wider class of Kac potentials. Both the paper of LP and the present one are motivated to some extent by the work of van Kampen [8].

19 Commun math Phys., Vol 15 
The main result of LP was to prove rigorously, for a certain class of Kac potentials (including, for example, non-positive Kac potentials), that the free energy density $a(\varrho, \gamma)$ of a system with the potential (1.1) and density $\varrho$ has the limit

$$
a(\varrho, 0+) \equiv \lim _{\gamma \rightarrow 0} a(\varrho, \gamma)=C E\left[a^{0}(\varrho)+\frac{1}{2} \alpha \varrho^{2}\right]
$$

where ${ }^{1} \alpha \equiv \int d \mathbf{s} K(\mathbf{s}), a^{0}(\varrho)$ is the free energy density of a system (called the reference system) with two-body potential $q(\mathbf{r})$, and $C E f(\varrho)$, called the convex envelope of the function $f(\varrho)$, is defined for arbitrary $f$ as the maximal convex function not exceeding $f(\varrho)$. They deduced that the limit $\pi(\varrho, 0+)$ of the pressure $\pi(\varrho, \gamma) \equiv \varrho \partial / \partial \varrho a(\varrho, \gamma)-a(\varrho, \gamma)$ is given by the Maxwell construction (or equal area rule) [4] applied to the function

$$
\pi^{0}(\varrho)+\frac{1}{2} \alpha \varrho^{2}
$$

where $\pi^{0}(\varrho)$ is the pressure of the reference system. This strongly resembles Maxwell's modification of the van der Waals equation of state, and is identical to it in the one dimensional case if the reference system consists of hard rods [3]. Consequently, the limit $\gamma \rightarrow 0$ is known as the van der Waals limit.

The result (1.2) can also be formulated in terms of the pressure $p(\mu, \gamma)$ expressed as a function of the chemical potential $\mu$. We then find

$$
p(\mu, 0+) \equiv \lim _{\gamma \rightarrow 0} p(\mu, \gamma)=\max _{\varrho}\left[\mu \varrho-a^{0}(\varrho)-\frac{1}{2} \alpha \varrho^{2}\right] .
$$

To deduce this from (1.2) we use the general relationship ${ }^{2}$

$$
p(\mu, \gamma)=\max _{\varrho}[\mu \varrho-a(\varrho, \gamma)]
$$

and note (as can be seen from Fig. 1) that

$$
\max _{\varrho}[\mu \varrho-C E f(\varrho)]=\sup _{\varrho}[\mu \varrho-f(\varrho)]
$$

for any function $f$. Now taking the limit $\gamma \rightarrow 0$ of (1.5) and using (1.2) and (1.6) we obtain (1.4). The interchange of the maximum and the limit is justified ${ }^{2}$ by the fact $[4]$ that $a(\varrho, \gamma)$ tends uniformly to its limit on a suitable interval of values of $\varrho$. Alternatively Eq. (1.4) can be deduced from first principles by using the method of LP in the grand ensemble.

\footnotetext{
1 When the range of an integral is not specified, it is over all of $v$-dimensional space.

${ }^{2}$ See Ref. [10], Section 10.
} 


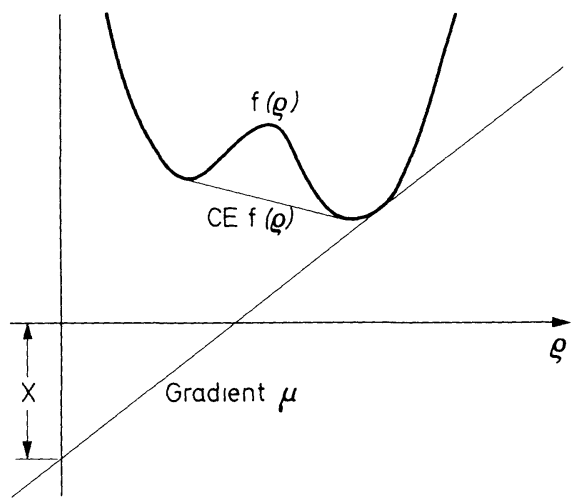

Fig. 1. Both sides of (1.6) are equal to the distance $x$

For a more general class of Kac potentials, LP obtained upper and lower bounds on $a(\varrho, 0+)$, but they did not prove for these potentials that the limits $a(\varrho, 0+)$ and $p(\mu, 0+)$ exist. In the present paper we provide such proofs and obtain expressions for the limit functions. We also include the effects of an external potential of the form $\psi(\gamma \mathbf{x})$.

\section{Definitions, Results, and Outline of Method}

We confine our attention for the moment to the pressure $p(\mu, \gamma)$, since the method and the main result are considerably simpler for this function. The free energy will be dealt with in Section VI.

The function $p(\mu, \gamma)$, called the pressure in the grand ensemble, can be defined by the thermodynamic limit $[10]$

$$
p(\mu, \gamma) \equiv \lim _{|\Omega| \rightarrow \infty} P(\mu, \Omega, \gamma)
$$

where $\Omega$ is a cube of volume $|\Omega|$ and

$$
P(\mu, \Omega, \gamma) \equiv \frac{1}{\beta|\Omega|} \log \Xi(\mu, \Omega, \gamma) .
$$

$\beta$ being the reciprocal temperature and $\Xi$ the grand partition function, defined by

$$
\Xi(\mu, \Omega, \gamma) \equiv \sum_{N=0}^{\infty} e^{\beta \mu N} Z(N, \Omega, \gamma)
$$

Here, $Z(N, \Omega, \gamma)$ is the partition function for $N$ particles in $\Omega$, defined for $N=0$ by $Z(0, \Omega, \gamma) \equiv 1$ and for $N \geqq 1$ by

$$
Z(N, \Omega, \gamma) \equiv \frac{1}{N ! \Lambda^{v N}} \int_{\Omega} d \mathbf{x}_{1} \ldots \int_{\Omega} d \mathbf{x}_{N} \exp \left(-\beta V_{N}\right)
$$


where $A$ is the thermal wavelength $[10], V_{1} \equiv \psi\left(\gamma \mathbf{x}_{1}\right)$ and

$$
V_{N} \equiv \sum_{1 \leqq a<b \leqq N} v\left(\mathbf{x}_{a}-\mathbf{x}_{b}, \gamma\right)+\sum_{1 \leqq a \leqq N} \psi\left(\gamma \mathbf{x}_{a}\right)
$$

for $N \geqq 2$, with $v(\mathbf{r}, \gamma)$ defined by (1.1) and $\psi(\gamma \mathbf{x})$ a periodic external potential whose period is proportional to $\gamma^{-1}$. The potential $\psi$ provides, among other things, a way of calculating the correlation functions in the van der Waals limit by functional differentiation; we hope to present this calculation in a future publication.

The conditions to be satisfied by the interaction potentials $q$ and $K$ are

$$
\begin{aligned}
& q(\mathbf{r})=q(-\mathbf{r}), \quad K(\mathbf{s})=K(-\mathbf{s}), \\
& \left.\begin{array}{c}
q(\mathbf{r})=\infty \text { for }|\mathbf{r}|<r_{0} \\
|q(\mathbf{r})|<C|\mathbf{r}|^{-v-\varepsilon} \text { for }|\mathbf{r}| \geqq r_{0}
\end{array}\right\} \\
& |K(\mathbf{s})|<k(|\mathbf{s}|)<\bar{K} \text { for all } \mathbf{s},
\end{aligned}
$$

$K$ is Riemann integrable over any any bounded region of $v$-dimensional space,

where $\bar{K}, C, r_{0}$ and $\varepsilon$ are positive constants, and $k(t)$ is a non-increasing function such that $\int d \mathbf{s} k(|\mathbf{s}|)<\infty$. Condition (2.7) implies that the particles have a $v$-dimensional spherical hard core of diameter $r_{0}$. The function $\psi$ satisfies the conditions

$\psi(\mathbf{y})$ is periodic over an infinite cubic lattice with unit cell $J$,

$|\psi(\mathbf{y})|<\bar{\psi}$, a positive constant, for all $\mathbf{y}$,

$\psi$ is Riemann integrable over any bounded region of $v$-dimensional space.

In the case $\psi=0$ it was shown by Dobrushin [9] that the conditions $(2.6,7,8)$ ensure the existence of $p(\mu, \gamma)$. It is not difficult to extend the argument of Dobrushin and prove that the thermodynamic limit also exists if a periodic external potential, bounded below, is present. We shall not give this proof here.

Our first result is

Theorem 1. Under conditions (2.6-12), the van der Waals limit $p(\mu, 0+)$ of the pressure $p(\mu, \gamma)$ exists and is given by

$$
p(\mu, 0+)=\lim _{|D| \rightarrow \infty} \sup _{n \in \mathscr{R}} F(n, \mu, D)
$$

or equivalently by

$$
p(\mu, 0+)=\sup _{n \in \mathscr{R}} F(n, \mu, \infty)
$$


The convergence of $p(\mu, \gamma)$ to $p(\mu, 0+)$ is uniform on any closed interval of values of $\mu$. The functional $F(n, \mu, D)$ is defined to be

$$
\begin{aligned}
\frac{1}{|D|} \int_{D} d \mathbf{y}\left\{\mu n(\mathbf{y})-\psi(\mathbf{y}) n(\mathbf{y})-a^{0}[n(\mathbf{y})]\right\} \\
-\frac{1}{2|D|} \int_{D} d \mathbf{y} \int_{D} d \mathbf{y}^{\prime} n(\mathbf{y}) n\left(\mathbf{y}^{\prime}\right) K\left(\mathbf{y}-\mathbf{y}^{\prime}\right)
\end{aligned}
$$

and $F(n, \mu, \infty)$ is defined by

$$
F(n, \mu, \infty) \equiv \lim _{|D| \rightarrow \infty} F(n, \mu, D)
$$

The limits in (2.13) and (2.16) are taken over any ascending ${ }^{3}$ sequence of $v$-dimensional cubes $D$ (of volume $|D|$ ) whose sides are multiples of and are parallel to those of $J$. The set $\mathscr{R}$ comprises all functions $n$ which are Riemann integrable on every bounded region of $v$-dimensional space, satisfy $0 \leqq n(\mathbf{y}) \leqq \varrho_{c}$ where $\varrho_{c}$ is the close packing density [13] for spheres of diameter $r_{0}$, and are periodic ${ }^{4}$, with a unit cell that need not be $J$.

All the integrals in (2.15), and henceforth, are taken as Lebesgue integrals. The formal similarity between the result (2.14) (in the case $\psi=0$ ) and the equation of state (1.4) is apparent. In particular they are identical if there is a function $n$ independent of $\mathbf{y}$ which maximizes $F(n, \mu, \infty)$.

Our method of proof of Theorem 1 is similar to the method used by LP. Starting from (2.4), we divide the cube $\Omega$ into a number of cells of volume $\omega$, obtain upper and lower bounds on $P(\mu, \gamma, \Omega)$ in terms of $\Omega, \gamma$ and $\omega$, and then take the succession of limits (called the LP triple limit)

$$
\text { first }|\Omega| \rightarrow \infty \text {, then } \gamma \rightarrow 0 \text { and finally } \omega \rightarrow \infty
$$

of these bounds. We also use an additional operation which consists of restricting the density $\varrho$ of the particles thus:

$$
\varrho \leqq \varrho^{\prime} \quad \text { where } \varrho^{\prime}<\varrho_{c},
$$

and finally taking the limit $\varrho^{\prime} \rightarrow \varrho_{c}$ after the LP triple limit. When this sequence of limits has been taken, the upper and lower bounds coincide and are given by (2.14) or (2.13).

\footnotetext{
${ }^{3}$ A sequence of sets $E_{1}, E_{2}, E_{3} \ldots$ is ascending if $E_{1} \subset E_{2} \subset E_{3} \ldots$.

${ }^{4}$ I.e. we can find $v$ linearly independent vectors $\mathbf{k}_{1} \ldots \mathbf{k}_{v}$ such that $n\left(\mathbf{y}+\mathbf{k}_{\lambda}\right)=n(\mathbf{y})$ for $\lambda=1 \ldots v$ and for all $\mathbf{y}$.
} 


\section{Properties of $F(n, \mu, D)$ and $F(n, \mu, \infty)$}

Before proving Theorem 1, we derive some properties of the functionals defined therein. We prove firstly that $F(n, D)$ and $F(n, \infty)$ exist $^{5}$ (or have value $-\infty$ ), and that they both have a supremum for $n \in \mathscr{R}$. Secondly we prove the equivalence of (2.13) and (2.14). Finally, for later use in the proof of Theorem 1 , we show that $F(n, D)$ is continuous with respect to $n$ in a certain sense.

To show that $F(n, D)$ exists, we note from (2.15) that it is the sum of four integrals. The first, second and last integrals exist because products of integrable functions are integrable. To study the third integral we define, for any $\varrho^{\prime}$ satisfying $0<\varrho^{\prime}<\varrho_{c}$,

$$
a_{\varrho^{\prime}}^{0}(\varrho) \equiv\left\{\begin{array}{lll}
a^{0}(\varrho) & \text { for } & 0 \leqq \varrho \leqq \varrho^{\prime} \\
a^{0}\left(\varrho^{\prime}\right) & \text { for } & \varrho^{\prime} \leqq \varrho \leqq \varrho_{c}
\end{array} .\right.
$$

Since $a_{\varrho^{\prime}}^{0}(\varrho)$ is a uniformly continuous function [10] of $\varrho$ in the closed interval $\left[0, \varrho_{c}\right]$, it follows $[11]$ that $a_{\varrho^{\prime}}^{0}[n(\mathbf{y})]$ is measurable. Consider, for a given $n$, the sequence of functions

$$
f_{\varrho^{\prime}}(\mathbf{y}) \equiv a_{\varrho^{\prime}}^{0}[n(\mathbf{y})]-a^{0}\left(\varrho_{0}\right)
$$

where $\varrho_{0}$ is a value of $\varrho$ where $a^{0}(\varrho)$ attains its minimum. The functions $f_{Q^{\prime}}$ are non-negative, measurable and, since $a^{0}$ is convex, form a nondecreasing (as $\varrho^{\prime}$ increases) sequence for $\varrho^{\prime} \geqq \varrho_{0}$. Now defining

$$
f(\mathbf{y}) \equiv \lim _{\varrho^{\prime} \rightarrow \varrho_{c}} f_{\varrho^{\prime}}(\mathbf{y})=a^{0}[n(\mathbf{y})]-a^{0}\left(\varrho_{0}\right)
$$

we deduce from Lebesgue's monotone convergence theorem [11] that $\int_{D} d \mathbf{y} f(\mathbf{y})$ either exists or is infinite, and hence that $\int_{D} d \mathbf{y} a^{0}[n(\mathbf{y})]$ either exists or is infinite. We have proved that $F(n, D)$ either exists or is equal to $-\infty$ (depending on the choice of $n$ ) for all $n \in \mathscr{R}$. In particular, $F$ is finite if $n(\mathbf{y})$ is bounded away from $\varrho_{c}$.

Next we show that $F(n, \infty)$ exists, and if $n$ has the unit cell $\Gamma$

$$
\begin{aligned}
F(n, \infty)= & \frac{1}{|\Gamma|} \int_{\Gamma} d \mathbf{y}\left\{\mu n(\mathbf{y})-a^{0}[n(\mathbf{y})]-\frac{1}{2} n(\mathbf{y}) \int d \mathbf{y}^{\prime} n\left(\mathbf{y}^{\prime}\right) K\left(\mathbf{y}-\mathbf{y}^{\prime}\right)\right\} \\
& -\lim _{|D| \rightarrow \infty} \frac{1}{|D|} \int_{D} d \mathbf{y} n(\mathbf{y}) \psi(\mathbf{y}) .
\end{aligned}
$$

\footnotetext{
${ }^{5}$ We omit the dependence of $F$ on $\mu$ in this section.
} 
To prove this we note firstly that the limit on the right side exists because of the periodicity of $n$ and $\psi$. Secondly, we consider the difference

$$
\begin{aligned}
& \delta(D) \equiv \frac{1}{|\Gamma|} \int_{\Gamma} d \mathbf{y} n(\mathbf{y}) \int d \mathbf{y}^{\prime} n\left(\mathbf{y}^{\prime}\right) K\left(\mathbf{y}-\mathbf{y}^{\prime}\right) \\
&-\frac{1}{|D|} \int_{D} d \mathbf{y} n(\mathbf{y}) \int_{D} d \mathbf{y}^{\prime} n\left(\mathbf{y}^{\prime}\right) K\left(\mathbf{y}-\mathbf{y}^{\prime}\right)
\end{aligned}
$$

between the quadratic terms in the expressions (2.15) and (3.4) for $F(n, \infty)$. Now $D$ can be expressed as a union of regions congruent to the unit cell $\Gamma$ together with a region $\sigma$ whose volume $|\sigma|$ is of order $|D|^{1-1 / v}$ (proportional to the surface area of $D$ ). Hence we can, by (2.8) and the periodicity of $n$, replace $\Gamma$ by $D$ in the first term on the right side of (3.5) if we add a correction of order $|D|^{-1 / v}$. This correction has the upper bound $\varrho_{c}^{2}|\sigma||D|^{-1} \int d \mathbf{s}|K(\mathbf{s})|$. We now have

$$
\begin{aligned}
|\delta(D)| & =\left|\frac{1}{|D|} \int_{D} d \mathbf{y} n(\mathbf{y}) \int_{D^{c}} d \mathbf{y}^{\prime} n\left(\mathbf{y}^{\prime}\right) K\left(\mathbf{y}-\mathbf{y}^{\prime}\right)\right|+0\left(|D|^{-1 / v}\right) \\
& \leqq \varrho_{c}^{2} \frac{1}{|D|} \int_{D} d \mathbf{y} \int_{D^{c}} d \mathbf{y}^{\prime}\left|K\left(\mathbf{y}-\mathbf{y}^{\prime}\right)\right|+0\left(|D|^{-1 / v}\right)
\end{aligned}
$$

where $D^{c}$ is the complement of $D$. By the argument applied by LP to their Eq. (2.15), the first term on the right side vanishes when $|D| \rightarrow \infty$. Thirdly, we note that the terms in (2.15) involving $\mu n$ and $a^{0}(n)$ tend to the corresponding terms in (3.4) because of the periodicity of $n$. This completes the proof of (3.4).

The fact that $F(n, D)$ and $F(n, \infty)$ have suprema follows from the inequality

$$
F(n, D) \leqq \varrho_{c}|\mu|+\varrho_{c} \bar{\psi}-a^{0}\left(\varrho_{0}\right)+\frac{1}{2} \varrho_{c}^{2} \int d \mathbf{s}|K(\mathbf{s})|,
$$

which in turn follows from (2.15) and (2.11).

To prove the equivalence of (2.13) and (2.14) we need

Lemma 1. If $\mathscr{R}_{\Gamma}$ is the subclass of $\mathscr{R}$ consisting of functions with unit cell $\Gamma$, and $\Gamma$ is a cube whose sides are parallel to and are multiples of those of $J$ (the unit cell of $\psi$ ), then we can find an $\varepsilon(\Gamma)>0$ such that $\varepsilon(\Gamma) \rightarrow 0$ as $|\Gamma| \rightarrow \infty$ and

$$
|F(n, \Gamma)-F(n, \infty)|<\varepsilon(\Gamma)
$$

for all $n \in \mathscr{R}_{\Gamma}$. Here $|\Gamma|$ means the volume of $\Gamma$. 
Proof. The terms involving $\mu n$ and $a^{0}(n)$ in the expression (2.15) for $F(n, \Gamma)$ are identical to the corresponding terms in the expression (3.4) for $F(n, \infty)$. By an argument like that leading to (3.6), we deduce that difference between the quadratic terms in $F(n, \Gamma)$ and in $F(n, \infty)$ is bounded by an amount $\varepsilon(\Gamma)$ independent of $n$ and tending to zero as $|\Gamma| \rightarrow \infty$. Also, because $n(\mathbf{y}) \psi(\mathbf{y})$ has unit cell $\Gamma$, we deduce that

$$
\lim _{|D| \rightarrow \infty} \frac{1}{|D|} \int_{D} d \mathbf{y} n(\mathbf{y}) \psi(\mathbf{y})=\frac{1}{|\Gamma|} \int_{\Gamma} d \mathbf{y} n(\mathbf{y}) \psi(\mathbf{y}),
$$

i.e. the terms involving $\psi$ in $F(n, \Gamma)$ and $F(n, \infty)$ are equal. This completes the proof of (3.8).

It follows from (3.8) that

$$
\sup _{n \in \mathscr{R}_{\Gamma}} F(n, \Gamma) \leqq \sup _{n \in \mathscr{R}_{\Gamma}} F(n, \infty)+\varepsilon(\Gamma) .
$$

We can replace $\mathscr{R}_{\Gamma}$ by $\mathscr{R}$ on the left side because $F(n, \Gamma)$ depends only on the values of $n(\mathbf{y})$ for $\mathbf{y}$ in $\Gamma$, and on the right side because $\mathscr{R}_{\Gamma} \subset \mathscr{R}$. Taking the limit $|\Gamma| \rightarrow \infty$ of the resulting inequality gives

$$
\limsup _{|D| \rightarrow \infty} \sup _{n \in \mathscr{R}} F(n, D) \leqq \sup _{n \in \mathscr{R}} F(n, \infty)
$$

provided that every $D$ is related to $J$ as specified in Theorem 1 . The reverse of this inequality also holds because $F(n, \infty) \equiv \lim _{D} F(n, D)$ $\leqq \liminf _{D} \sup _{n} F(n, D)$ for all $n$. This proves the equivalence of (2.13) and (2.14) and the existence of the limit on the right side of (2.13).

Finally, we show that the functional $F(n, D)$ is continuous in $n$. For any $\varrho^{\prime}$ satisfying $0<\varrho^{\prime}<\varrho_{c}$, let $\mathscr{R}\left(\varrho^{\prime}\right)$ be the subclass of $\mathscr{R}$ consisting of functions $n$ which are bounded above by $\varrho^{\prime}$. We then have

Lemma 2. The functional $F(n, D)$ is continuous with respect to $n$, for $n \in \mathscr{R}\left(\varrho^{\prime}\right)$, in the following sense: for any $\varepsilon>0$ we can find a $\delta>0$ such that, for $n_{1} \in \mathscr{R}\left(\varrho^{\prime}\right)$ and $n_{2} \in \mathscr{R}\left(\varrho^{\prime}\right)$,

implies

$$
\frac{1}{|D|} \int_{D} d \mathbf{y}\left|n_{1}(\mathbf{y})-n_{2}(\mathbf{y})\right|<\delta
$$

$$
\left|F\left(n_{1}, D\right)-F\left(n_{2}, D\right)\right|<\varepsilon .
$$

Further, the continuity is uniform in both $n$ and $D$. (In the language of modern analysis, $F$ is continuous on a subspace of the metric space $L_{1}$.)

Proof. From (3.12) we have

$$
\left|\frac{1}{|D|} \int_{D} d \mathbf{y}\left[n_{2}(\mathbf{y})-n_{1}(\mathbf{y})\right] \mu\right|<|\mu| \delta
$$


and from (2.11)

$$
\left|\frac{1}{|D|} \int_{D} d \mathbf{y}\left[n_{2}(\mathbf{y})-n_{1}(\mathbf{y})\right] \psi(\mathbf{y})\right|<\bar{\psi} \delta
$$

We also have

$$
\begin{aligned}
& n_{2}(\mathbf{y}) n_{2}\left(\mathbf{y}^{\prime}\right)-n_{1}(\mathbf{y}) n_{1}\left(\mathbf{y}^{\prime}\right) \\
& \quad=\left[n_{2}(\mathbf{y})-n_{1}(\mathbf{y})\right]\left[n_{2}\left(\mathbf{y}^{\prime}\right)+n_{1}\left(\mathbf{y}^{\prime}\right)\right]+n_{1}(\mathbf{y}) n_{2}\left(\mathbf{y}^{\prime}\right)-n_{1}\left(\mathbf{y}^{\prime}\right) n_{2}(\mathbf{y}) .
\end{aligned}
$$

Multiplying by $K\left(\mathbf{y}-\mathbf{y}^{\prime}\right)$ and integrating we find, since $n_{1}(\mathbf{y})+n_{2}(\mathbf{y})<2 \varrho_{c}$, that

$$
\begin{aligned}
\left|\frac{1}{2|D|} \int_{D} d \mathbf{y} \int_{D} d \mathbf{y}^{\prime} K\left(\mathbf{y}-\mathbf{y}^{\prime}\right)\left[n_{2}(\mathbf{y}) n_{2}\left(\mathbf{y}^{\prime}\right)-n_{1}(\mathbf{y}) n_{1}\left(\mathbf{y}^{\prime}\right)\right]\right| \\
<\frac{\varrho_{c}}{|D|} \int_{D} d \mathbf{y}\left|n_{2}(\mathbf{y})-n_{1}(\mathbf{y})\right| \int_{D} d \mathbf{y}^{\prime}\left|K\left(\mathbf{y}-\mathbf{y}^{\prime}\right)\right| \\
<\varrho_{c} \delta \int d \mathbf{s}|K(\mathbf{s})| .
\end{aligned}
$$

Since $a^{0}(\varrho)$ is uniformly continuous in the closed interval $\left[0, \varrho^{\prime}\right]$, it follows that for the given $\delta$ we can find a positive number $\sigma(\delta)$ such that (i) for all $\varrho_{1}$ and $\varrho_{2}$ in $\left[0, \varrho^{\prime}\right],\left|\varrho_{1}-\varrho_{2}\right|<\sqrt{\delta}$ implies $\left|a^{0}\left(\varrho_{1}\right)-a^{0}\left(\varrho_{2}\right)\right|<\sigma(\delta)$, and (ii) $\sigma(\delta) \rightarrow 0$ as $\delta \rightarrow 0$. For a given $n_{1}$ and $n_{2}$, we can express $D$ as the sum of two parts $D_{<}$and $D_{>}$, where $\left|n_{1}-n_{2}\right|<\sqrt{\delta}$ for $\mathbf{y} \in D_{<}$and $\left|n_{1}-n_{2}\right| \geqq \sqrt{\delta}$ for $\mathbf{y} \in D_{>}$. Then we have

where

$$
\left|a^{0}\left[n_{1}(\mathbf{y})\right]-a^{0}\left[n_{2}(\mathbf{y})\right]\right| \leqq\left\{\begin{array}{l}
\sigma(\delta) \text { for } \mathbf{y} \in D_{<} \\
2 S \text { for } \mathbf{y} \in D_{>},
\end{array}\right.
$$

But (3.12) implies

$$
S \equiv \sup _{0 \leqq \varrho \leqq \varrho^{\prime}}\left|a^{0}(\varrho)\right|
$$

$$
\left|D_{>}\right| \sqrt{\delta}<\int_{D>} d \mathbf{y}\left|n_{1}-n_{2}\right| \leqq \int_{D} d \mathbf{y}\left|n_{1}-n_{2}\right|<|D| \delta
$$

so that $\left|D_{>}\right|<|D| \sqrt{\delta}$. It follows from this and (3.18) that

$$
\int_{D} d \mathbf{y}\left|a^{0}\left[n_{1}(\mathbf{y})\right]-a^{0}\left[n_{2}(\mathbf{y})\right]\right| \leqq[\sigma(\delta)+2 S \sqrt{\delta}]|D| .
$$

Finally, from $(3.14,15,17$, and 21) we have

$$
\begin{aligned}
\mid F\left(n_{1}, D\right) & -F\left(n_{2}, D\right) \mid \\
& <|\mu| \delta+\bar{\psi} \delta+\varrho_{c} \delta \int d \mathbf{s}|K(\mathbf{s})|+\sigma(\delta)+2 S \sqrt{\delta} \rightarrow 0 \quad \text { as } \quad \delta \rightarrow 0,
\end{aligned}
$$

which proves the lemma ${ }^{6}$.

\footnotetext{
${ }^{6}$ The same argument proves that $F(n, D)$ is upper semi-continuous for $n \in \mathscr{R}$.
} 


\section{Lower Bound on the Pressure}

The main part or the proof of Theorem 1 now consists of proving the statement (2.13). To do this we show in the present section that the right side of (2.13) is a lower bound on $p(\mu, 0+)$, and in the following section that it is an upper bound.

Finding a lower bound on $P(\mu, \Omega, \gamma)$ is equivalent to finding a lower bound on the partition function $Z(N, \Omega, \gamma)$. To obtain such a lower bound, we follow LP and divide $\Omega$ into $M$ smaller cubical regions $\omega_{1} \ldots \omega_{M}$, each of volume $\omega$, so that $|\Omega|=M \omega$. We shall find a succession of lower bounds, firstly in terms of the occupation numbers of these cells, secondly in terms of step functions and finally in terms of functions in $\mathscr{R}$. Let $\omega_{i}^{\prime}$ be a subcube of $\omega_{i}$, concentric with and similarly oriented to $\omega_{i}$ and of volume $\omega^{\prime}$ where $\omega^{\prime}<\omega$. Now, following LP, we obtain from the definition (2.4)

$$
Z(N, \Omega, \gamma)>\frac{\Lambda^{-v N}}{N_{1} ! \ldots N_{M} !} \int_{\left(\omega_{1}^{\prime}\right)^{N_{1}}} \cdots \int_{\left(\omega_{M}^{\prime}\right)^{N_{M}}} d \mathbf{x}_{1} \ldots d \mathbf{x}_{N} e^{-\beta V_{N}}
$$

where $N_{1} \ldots N_{M}$ is any set of integers whose sum is $N$ and which satisfy

$$
O \leqq N_{i} \leqq N_{c}\left(\omega^{\prime}\right)
$$

where $N_{c}\left(\omega^{\prime}\right)$ is the maximum number of spheres of diameter $r_{0}$ whose centres can be contained by one cube $\omega_{i}^{\prime}$. The notation in (4.1) indicates that the first $N_{1}$ volume integrations are over $\omega_{1}^{\prime}$, the next $N_{2}$ over $\omega_{2}^{\prime}$, and so on. It follows that

$$
Z(N, \Omega, \gamma)>\left[\prod_{i=1}^{M} Z^{0}\left(N_{i}, \omega^{\prime}\right)\right] \exp \left(-\beta Q_{\max }^{\prime \prime}-\beta W_{\max }\right)
$$

where $Z^{0}$ is the partition function of the reference system; $Q_{\max }^{\prime \prime}$ is an upper bound, for all $\mathbf{x}_{1} \ldots \mathbf{x}_{N}$ and all $N$, on the total energy of interaction due to $q(\mathbf{r})$ resulting from pairs of particles that are in different cells $\omega_{i}^{\prime}$; and $W_{\max }\left(N_{1} \ldots N_{M}\right)$ is an upper bound on the total potential energy due to $\gamma^{v} K(\gamma \mathbf{r})$ and $\psi(\gamma \mathbf{x})$ for configurations specified by the integral in (4.1).

To estimate $W_{\max }$ we define

$$
\psi_{i}^{+} \equiv \sup _{\mathbf{x} \in \omega_{1}} \psi(\gamma \mathbf{x}) \geqq \sup _{\mathbf{x} \in \omega_{i}^{\prime}} \psi(\gamma \mathbf{x}) .
$$

Then $\psi$ contributes $\sum_{i} N_{i} \psi_{i}^{+}$to $W_{\max }$. Next define

$$
K_{i j}^{+} \equiv \sup _{\mathbf{x} \in \omega_{l}, \mathbf{x}^{\prime} \in \omega_{J}} K\left(\gamma \mathbf{x}-\gamma \mathbf{x}^{\prime}\right) .
$$


Then $K$ contributes to $W_{\max }$ the term $\frac{1}{2} \gamma^{v} \sum_{i \neq j} N_{i} N_{j} K_{i j}^{+}$due to particles in different cells $\omega_{i}^{\prime}$, and the term $\frac{1}{2} \gamma^{v} \sum_{i} N_{i}\left(N_{i}-1\right) \mathrm{K}_{i i}^{+}$due to particles in the same cell. Consequently we have

$$
W_{\max }=\frac{1}{2} \gamma^{\nu} \sum_{i}^{M} \sum_{j}^{M} N_{i} N_{j} K_{i j}^{+}+\sum_{i}^{M}\left(N_{i} \psi_{i}^{+}-\frac{1}{2} \gamma^{v} N_{i} K_{i i}^{+}\right) .
$$

The grand partition function, defined by (2.3), has a lower bound given by

$$
\Xi(\mu, \Omega, \gamma)>\exp (\beta \mu N) \cdot Z(N, \Omega, \gamma)
$$

for any $N$. It follows from $(4.3,6$ and 7$)$ that the pressure $P$, defined by (2.2), has a lower bound given by

$$
\begin{aligned}
& P(\mu, \Omega, \gamma)>-Q_{\text {max }}^{\prime \prime} /|\Omega|-\frac{1}{2} \gamma^{v}|\Omega|^{-1} \sum_{i j} N_{i} N_{j} K_{i j}^{+} \\
& +|\Omega|^{-1} \sum_{i}\left[N_{i} \mu-N_{i} \psi_{i}^{+}+\beta^{-1} \log Z^{0}\left(N_{i}, \omega^{\prime}\right)+\frac{1}{2} \gamma^{v} N_{i} K_{i i}^{+}\right]
\end{aligned}
$$

for any set of integers $0 \leqq N_{i} \leqq N_{c}\left(\omega^{\prime}\right)$. (Their sum is now arbitrary.)

The quantity $Q_{\max }^{\prime \prime} /|\Omega| \equiv \varepsilon_{1}$, say, vanishes in the LP triple limit (2.17) for a suitable choice of $\omega^{\prime}$, as shown by LP ${ }^{7}$. Also the final term in (4.8) satisfies

$$
\frac{1}{2} \gamma^{v} \sum_{i} N_{i} K_{i i}^{+} /|\Omega| \geqq-\frac{1}{2} \gamma^{\nu} \bar{K} N_{c}(\omega) / \omega \equiv-\varepsilon_{2}, \quad \text { say },
$$

from (2.8). Then $\varepsilon_{2}$ vanishes in the LP triple limit.

It is convenient to replace $K_{i j}^{+}$in (4.8) by

$$
K_{i j}^{-} \equiv \inf _{\mathbf{x} \in \omega_{l}, \mathbf{x}^{\prime} \in \omega_{j}} K\left(\gamma \mathbf{x}-\gamma \mathbf{x}^{\prime}\right)
$$

We can make this replacement if we also subtract from the right side of (4.8) the correction term

$$
\begin{aligned}
\frac{1}{2} \gamma^{v}|\Omega|^{-1} & \sum_{i j} N_{i} N_{j}\left(K_{i j}^{+}-K_{i j}^{-}\right) \\
& \leqq\left[N_{c}(\omega) / \omega\right]^{2} \sum_{i}^{\infty} \gamma^{v} \omega\left(K_{i j}^{+}-K_{i j}^{-}\right) \equiv \varepsilon_{3}, \quad \text { say }
\end{aligned}
$$

${ }^{7}$ Since our $Q_{\max }^{\prime \prime}$ is an upper bound for all $N$, we must replace $\varrho$ by $\varrho_{c}$ in the estimate of this quantity given by LP. 
where the sum $\sum^{\infty}$ is over an infinite lattice of $\omega_{i}$ 's. But from (2.8) and (2.9), $\int d \mathbf{s} K(\mathbf{s})$ exists as a Riemann integral and is finite. Hence, as shown by LP,

$$
\lim _{\gamma \rightarrow 0} \sum_{i}^{\infty} \gamma^{v} \omega K_{i j}^{+}=\int d \mathbf{s} K(\mathbf{s}), \text { for all } j .
$$

It follows that $\varepsilon_{3}$ vanishes in the LP triple limit. Similarly we can replace $\psi_{i}^{+}$in (4.8) by

$$
\psi_{i}^{-} \equiv \inf _{\mathbf{x} \in \omega_{2}} \psi(\gamma \mathbf{x})
$$

if we subtract the correction term

$$
|\Omega|^{-1} \sum_{i}^{M} N_{i}\left(\psi_{i}^{+}-\psi_{i}^{-}\right) \leqq N_{c}(\omega)|\Omega|^{-1} \sum_{i}^{M}\left(\psi_{i}^{+}-\psi_{i}^{-}\right) \equiv \varepsilon_{4}, \quad \text { say } .
$$

From conditions $(2.10,11$ and 12$)$ we deduce that $\psi(\mathbf{y})$ is Riemann integrable over its unit cell $J$, and hence

$$
\lim _{\gamma \rightarrow 0} \lim _{|\Omega| \rightarrow \infty} \frac{1}{|\Omega|} \sum_{i}^{M} \psi_{i}^{ \pm} \omega=\frac{1}{|J|} \int_{J} d \mathbf{y} \psi(\mathbf{y}) .
$$

It follows that $\varepsilon_{4}$ vanishes in the LP triple limit. The inequality (4.8) now reduces to

$$
\begin{aligned}
P(\mu, \Omega, \gamma)> & |\Omega|^{-1} \sum_{i}\left[N_{i} \mu-N_{i} \psi_{i}^{-}-A^{0}\left(N_{i}, \omega^{\prime}\right)\right] \\
& -\frac{1}{2} \gamma^{\nu}|\Omega|^{-1} \sum_{i j} N_{i} N_{j} K_{i j}^{-}-\varepsilon_{1} \cdots-\varepsilon_{4}
\end{aligned}
$$

where $A^{0}$, the free energy of the reference system, is defined by

$$
A^{0}(N, \Omega) \equiv-\beta^{-1} \log Z^{0}(N, \Omega) .
$$

The next step is to replace $A^{0}\left(N_{i}, \omega^{\prime}\right)$ by $\omega a^{0}\left(N_{i} / \omega\right)$ plus a correction, where $a^{0}(\varrho) \equiv \lim _{|\Omega| \rightarrow \infty} A^{0}(\varrho|\Omega|, \Omega) /|\Omega|$. To do this, we note that (4.16) holds if we impose any additional restriction on the $N_{i}$ 's. In particular, it holds if we specify that $N_{i} \leqq \varrho^{\prime} \omega$ where $\varrho^{\prime}$ is chosen to satisfy $0<\varrho^{\prime}<\varrho_{c}$. But, if $\varrho$ lies in the closed interval $\left[0, \varrho^{\prime}\right]$ and $A^{0}(N, \Omega)$ is defined for nonintegral $N$ by linear interpolation [10], then the sequence of functions $A^{0}\left(\varrho \omega, \omega^{\prime}\right) / \omega$ converges uniformly in $\varrho$ to $a^{0}(\varrho)$ as $\omega \rightarrow \infty$ and $\omega / \omega^{\prime} \rightarrow 1$, [10]. Hence, we can find a positive function $\varepsilon_{5}\left(\omega, \omega^{\prime}\right)$, independent of $N_{i}$ (but depending on $\varrho^{\prime}$ ) such that

$$
A^{0}\left(N_{i}, \omega^{\prime}\right) / \omega<a^{0}\left(N_{i} / \omega\right)+\varepsilon_{5}\left(\omega, \omega^{\prime}\right)
$$

for all $N_{i} \leqq \varrho^{\prime} \omega$, where $\varepsilon_{5}\left(\omega, \omega^{\prime}\right) \rightarrow 0$ as $\omega \rightarrow \infty$ and $\omega / \omega^{\prime} \rightarrow 1$. It follows that $\varepsilon_{5}$ vanishes in the LP triple limit. We can now replace $A^{0}\left(N_{i}, \omega^{\prime}\right)$ in 
(4.16) by $a^{0}\left(N_{i} / \omega\right) \omega$, if we subtract the correction, $\varepsilon_{5}$ from the right hand side.

The next step is to replace the $N_{i}$ 's in (4.16) by the step function

$$
n_{\text {step }}(\mathbf{y}) \equiv N_{i} / \omega \quad \text { for } \quad \mathbf{y} \in \bar{\omega}_{i} \text { and all } i
$$

where $\bar{\omega}_{i}$ is defined as the set of points $\mathbf{y}$ such that $\mathbf{y} / \gamma \in \omega_{i}$. Thus $\bar{\omega}_{i}$ is a cube of volume $\bar{\omega} \equiv \gamma^{v} \omega$. It follows from (4.10) and (4.13) that

and

$$
\psi_{i}^{-}=\inf _{\mathbf{y} \in \bar{\omega}_{\imath}} \psi(\mathbf{y})
$$

$$
K_{i j}^{-}=\inf _{\mathbf{y} \in \bar{\omega}_{t}, \mathbf{y}^{\prime} \in \bar{\omega}_{j}} K\left(\mathbf{y}-\mathbf{y}^{\prime}\right) .
$$

Let us also define $\bar{\Omega}$ as the set of points $\mathbf{y}$ such that $\mathbf{y} / \gamma \in \Omega$. The set $\bar{\Omega}$ is a cube of volume $\gamma^{v}|\Omega|$ and is filled by the cells $\bar{\omega}_{1} \ldots \bar{\omega}_{M}$. Now substituting (4.17-20) in (4.16) we obtain, for any $n_{\text {step }}$,

$$
P(\mu, \Omega, \gamma)>F\left(n_{\text {step }}, \bar{\Omega}\right)-\varepsilon_{1} \cdots-\varepsilon_{5}
$$

where $F$ is defined by $(2.15)$.

The next step is to use Lemma 2 and replace $n_{\text {step }}$ by a function $n^{\prime} \in \mathscr{R}\left(\varrho^{\prime}\right)$, where $\mathscr{R}\left(\varrho^{\prime}\right)$ was defined just before Lemma 2 . Firstly, for such an $n^{\prime}$, let us define the step function

$$
n_{\gamma}^{\prime}(\mathbf{y}) \equiv \frac{1}{\bar{\omega}} \int_{\bar{\omega}_{2}} d \mathbf{y} n^{\prime}(\mathbf{y}) \quad \text { for } \quad \mathbf{y} \in \bar{\omega}_{i} \quad \text { and all } i .
$$

We can find an $n_{\text {step }}$ such that

$$
\left|n_{\gamma}^{\prime}(\mathbf{y})-n_{\text {step }}(\mathbf{y})\right|<1 / \omega \text { for all } \mathbf{y} .
$$

Secondly, if $\Gamma$ is a unit cell of the periodic function $n^{\prime}$, we dissect the space of vectors $\mathbf{y}$ into an infinite lattice of cells $\Gamma_{\lambda}$ congruent to $\Gamma$. Then we have

$$
\frac{1}{|\bar{\Omega}|} \int_{\bar{\Omega}} d \mathbf{y}\left|n^{\prime}(\mathbf{y})-n_{\gamma}^{\prime}(\mathbf{y})\right|=\frac{1}{k} \sum_{\lambda=1}^{k} \frac{1}{|\Gamma|} \int_{\Gamma_{\lambda}} d \mathbf{y}\left|n^{\prime}(\mathbf{y})-n_{\gamma}^{\prime}(\mathbf{y})\right|+0\left(|\bar{\Omega}|^{-1 / v}\right)
$$

where $\Gamma_{1} \ldots \Gamma_{k}$ is the set of $\Gamma_{\lambda}$ 's which lie entirely inside $\bar{\Omega}$. Although $n^{\prime}$ is periodic over the $\Gamma_{\lambda}$ 's, $n_{\gamma}^{\prime}$ is not since each $\Gamma_{\lambda}$ is subdivided differently by the $\bar{\omega}_{i}$ 's. However, the theorem of Darboux [12] shows that

$$
\int_{\Gamma_{\lambda}} d \mathbf{y}\left|n^{\prime}-n_{\gamma}^{\prime}\right|<\delta(\bar{\omega}) \text { for all } \lambda,
$$

where $\delta(\bar{\omega})$ is independent of $\lambda$ and tends to zero as $\bar{\omega} \rightarrow 0$ and hence as $\gamma \rightarrow 0$. It follows from (4.24) that

$$
\lim _{\gamma \rightarrow 0} \limsup _{|\Omega| \rightarrow \infty} \frac{1}{|\bar{\Omega}|} \int_{\bar{\Omega}} d \mathbf{y}\left|n^{\prime}-n_{\gamma}^{\prime}\right|=0 .
$$


Combining this with (4.23), we deduce that for any $n^{\prime} \in \mathscr{R}\left(\varrho^{\prime}\right)$, we can find an $n_{\text {step }}(\mathbf{y})$ (which depends also on $\gamma$ and $\omega$ ) such that

$$
|\bar{\Omega}|^{-1} \int_{\bar{\Omega}} d \mathbf{y}\left|n^{\prime}-n_{\text {step }}\right|
$$

vanishes in the LP triple limit. Since $n_{\text {step }}$ belongs to $\mathscr{R}\left(\varrho^{\prime}\right)$, we deduce from Lemma 2 that

$$
\left|F\left(n^{\prime}, \bar{\Omega}\right)-F\left(n_{\text {step }}, \bar{\Omega}\right)\right|<\varepsilon_{6}
$$

where $\varepsilon_{6}$ vanishes in the LP triple limit. From (4.21) and (4.27) we conclude that for all $n^{\prime} \in \mathscr{R}\left(\varrho^{\prime}\right)$

$$
P(\mu, \Omega, \gamma)>F\left(n^{\prime}, \bar{\Omega}\right)-\varepsilon_{1} \cdots-\varepsilon_{6} .
$$

The next step is to show that $n^{\prime}$ may be replaced by any function $n \in \mathscr{R}$. For such an $n$, let us choose

$$
n^{\prime}(\mathbf{y}) \equiv\left\{\begin{array}{l}
n(\mathbf{y}) \text { where } 0 \leqq n(\mathbf{y}) \leqq \varrho^{\prime} \\
\varrho^{\prime} \text { elsewhere }
\end{array}\right.
$$

which clearly belongs to $\mathscr{R}\left(\varrho^{\prime}\right)$. Hence (4.28) holds for this $n^{\prime}$. Eq. (4.29) implies that $\left|n(\mathbf{y})-n^{\prime}(\mathbf{y})\right| \leqq \varrho_{c}-\varrho^{\prime}$ for all $\mathbf{y}$, and also, since $a^{0}(\varrho)$ is convex that $a^{0}\left[n^{\prime}(\mathbf{y})\right] \leqq a^{0}[n(\mathbf{y})]$ for $\varrho^{\prime} \geqq \varrho_{0}$, where $\varrho_{0}$ is a value of $\varrho$ where $a^{0}(\varrho)$ is minimum. Therefore, by an argument like that of Lemma 2 with $\delta$ replaced by $\varrho_{c}-\varrho^{\prime}$, we find that, provided $\varrho^{\prime} \geqq \varrho_{0}$,

where

$$
F\left(n^{\prime}, \bar{\Omega}\right) \geqq F(n, \bar{\Omega})-\Delta \quad \text { for all } \bar{\Omega},
$$

$$
\Delta \equiv\left[|\mu|+\bar{\psi}+\varrho_{c} \int d \mathbf{s}|K(\mathbf{s})|\right]\left(\varrho_{c}-\varrho^{\prime}\right) .
$$

Substituting (4.30) in (4.28) gives

$$
P(\mu, \Omega, \gamma) \geqq F(n, \bar{\Omega})-\varepsilon_{1} \cdots-\varepsilon_{6}-\Delta .
$$

Since this holds for all $n \in \mathscr{R}$, it follows that

$$
P(\mu, \Omega, \gamma) \geqq \sup _{n \in \mathscr{R}} F(n, \bar{\Omega})-\varepsilon_{1} \cdots-\varepsilon_{6}-\Delta .
$$

Finally, taking the LP triple limit followed by the limit $\varrho^{\prime} \rightarrow \varrho_{c}$ gives

$$
\liminf _{\gamma \rightarrow 0} p(\mu, \gamma) \geqq \lim _{|D| \rightarrow \infty} \sup _{n \in \mathscr{R}} F(n, \mu, D)
$$

where the right side was proved to exist in Section III. This takes us half way in our proof of the statement (2.13) of Theorem 1. 


\section{Upper Bound on the Pressure}

To find an upper bound on the pressure, we need an upper bound on $Z$. We use the same construction of cells $\omega_{i}$ as before and a similar method. Then, with the notation of (4.1), we have

$$
Z(N, \Omega, \gamma)<\sum_{N_{1} \ldots N_{M}}^{N} \frac{A^{-v N}}{N_{1} ! \ldots N_{M} !} \int_{\left(\omega_{1}\right)^{N_{1}}} \cdots \int_{\left(\omega_{M}\right)^{N_{M}}} d \mathbf{x}_{1} \ldots d \mathbf{x}_{N} e^{-\beta V_{N}}
$$

where the sum $\sum^{N}$ is over all integers $N_{i}$ whose sum is $N$ and which satisfy $0 \leqq N_{i} \leqq N_{c}(\omega)$. It follows that

$$
\begin{aligned}
& Z(N, \Omega, \gamma)< \exp \left(-\beta \tilde{Q}_{\min }\right) \sum_{N_{1} \ldots N_{M}}^{N}\left[\prod_{i=1}^{M} Z^{0}\left(N_{i}, \omega\right)\right] \\
& \cdot \exp \left[-\beta W_{\min }\left(N_{1} \ldots N_{N}\right)\right]
\end{aligned}
$$

where $\hat{Q}_{\min }$ is a lower bound, for all $\mathbf{x}_{1} \ldots \mathbf{x}_{N}$ and all $N$, on the total interaction energy due to $q(\mathbf{r})$ resulting from pairs of particles in different cells $\omega_{i}$. Also $W_{\min }$ is a lower bound on the potential energy due to $\gamma^{\nu} K(\gamma \mathbf{r})$ and $\psi(\gamma \mathbf{x})$ for configurations specified by $N_{1} \ldots N_{M}$. As in the derivation of (4.6) we deduce that

$$
W_{\min }=\frac{1}{2} \gamma^{v} \sum_{i j} N_{i} N_{j} K_{i j}^{-}+\sum_{i}\left(N_{i} \psi_{i}^{-}-\frac{1}{2} \gamma^{v} N_{i} K_{i i}^{-}\right)
$$

where $K_{i j}^{-}$and $\psi_{i}^{-}$are defined by (4.10) and (4.13).

It follows from (2.3) that

$$
\Xi(\mu, \Omega, \gamma)<e^{-\beta \tilde{Q}_{\min }} \sum_{N_{1} \ldots N_{M}}\left[\prod_{i} Z^{0}\left(N_{i}, \omega\right) e^{\beta \mu N_{i}}\right] e^{-\beta W_{\min }\left(N_{1} \ldots N_{M}\right)}
$$

where the restriction that the $N_{i}$ 's sum to $N$ no longer applies. Since $N_{i} \leqq N_{c}(\omega)$, there are at most $N_{c}(\omega)^{M}$ terms in the summation in (5.4), so that

$$
\Xi(\mu, \Omega, \gamma)<e^{-\beta \tilde{Q}_{\min }} N_{c}(\omega)^{M} \max _{N_{1} \ldots N_{M}}\left\{e^{-\beta W_{\min }} \prod_{i} e^{\beta \mu N_{i}} Z^{0}\left(N_{i}, \omega\right)\right\} .
$$

Then (2.2) gives

$$
\begin{aligned}
P(\mu, \Omega, \gamma)< & \frac{1}{|\Omega|} \max _{N_{1} \ldots N_{M}}\left\{\sum_{i}\left[N_{i} \mu-A^{0}\left(N_{i}, \omega\right)\right]-W_{\min }\right\} \\
& -\tilde{Q}_{\min } /|\Omega|-(\beta \omega)^{-1} \log N_{c}(\omega) .
\end{aligned}
$$

This inequality still holds if we allow the maximum to range over nonintegral values of the $N_{i}$, where $A^{0}\left(N_{i}, \omega\right)$ is defined by linear interpolation [10] for such $N_{i}$. 
The term $\hat{Q}_{\text {min }} /|\Omega| \equiv \varepsilon_{1}^{\prime}$ say, vanishes in the triple limit as shown by LP. The term $(\beta \omega)^{-1} \log N_{c}(\omega) \equiv \varepsilon_{2}^{\prime}$ say, also vanishes in this limit because, from LP, $1 \leqq N_{c}(\omega)<\varrho_{c}\left(\omega^{1 / v}+2 r_{0}\right)^{v}$. Put $\varepsilon_{3}^{\prime} \equiv \varepsilon_{2}, \varepsilon_{4}^{\prime} \equiv \varepsilon_{3}$ and $\varepsilon_{5}^{\prime} \equiv \varepsilon_{4}$, where $\varepsilon_{2}, \varepsilon_{3}$ and $\varepsilon_{4}$ were defined in the previous section and shown to vanish in the LP triple limit. Then (5.6) becomes

$$
\begin{aligned}
P(\mu, \Omega, \gamma)< & \frac{1}{|\Omega|} \max _{N_{1} \ldots N_{M}}\left\{\sum_{i}\left[N_{i} \mu-N_{i} \psi_{i}^{+}-A^{0}\left(N_{i}, \omega\right)\right]\right. \\
& \left.-\frac{1}{2} \gamma^{v} \sum_{i j} N_{i} N_{j} K_{i j}^{+}\right\}+\varepsilon_{1}^{\prime} \cdots+\varepsilon_{5}^{\prime}
\end{aligned}
$$

where we have replaced $\psi_{i}^{-}$and $K_{i j}^{-}$by $\psi_{i}^{+}$and $K_{i j}^{+}$, and added the appropriate correction terms.

The next step is to replace $A^{0}$ by $a^{0}$. To do this we first define

$$
f(N) \equiv\left\{\begin{array}{lll}
N & \text { if } & N \leqq \varrho^{\prime} \omega, \\
\varrho^{\prime} \omega & \text { if } & N \geqq \varrho^{\prime} \omega,
\end{array}\right.
$$

where $0<\varrho^{\prime}<\varrho_{c}$. Using the inequality of Penrose [13],

$$
Z^{0}(N+1, \omega) \leqq Z^{0}(N, \omega) \Lambda^{-v} e^{2 \beta \Phi^{\prime}}\left[\left(\omega^{1 / v}+2 r_{0}\right)^{v} / N-1 / \varrho_{c}\right],
$$

where $\Phi^{\prime}$ is a certain positive constant, we deduce that $A^{0}(N, \omega)>A^{0}\left(N^{\prime}, \omega\right)$ for all $\omega, N$ and $N^{\prime}$ such that $N>N^{\prime}>\varrho_{I}\left(\omega^{1 / v}+2 r_{0}\right)^{v}$, where

$$
\varrho_{I} \equiv\left[1 / \varrho_{c}+\Lambda^{v} e^{-2 \beta \Phi^{\prime}}\right]^{-1}<\varrho_{c} .
$$

Since $N_{i} \geqq f\left(N_{i}\right)$, it follows that

$$
A^{0}\left(N_{i}, \omega\right) \geqq A^{0}\left[f\left(N_{i}\right), \omega\right] \text { for } \varrho^{\prime} \geqq \varrho_{I}\left(1+2 r_{0} \omega^{-1 / v}\right)^{v} .
$$

Also, since $N_{i}-f\left(N_{i}\right) \leqq \omega\left(\varrho_{c}-\varrho^{\prime}\right)$, we deduce by an argument like that leading to (4.32) that

$$
\begin{aligned}
P(\mu, \Omega, \gamma)< & \frac{1}{|\Omega|} \max _{N_{1} \ldots N_{M}}\left\{\sum_{i}\left[f\left(N_{i}\right) \mu-f\left(N_{i}\right) \psi_{i}^{+}-A^{0}\left(f\left(N_{i}\right), \omega\right)\right]\right. \\
& \left.-\frac{1}{2} \gamma^{\nu} \sum_{i j} f\left(N_{i}\right) f\left(N_{j}\right) K_{i j}^{+}\right\}+\varepsilon_{1}^{\prime} \cdots+\varepsilon_{5}^{\prime}+\Delta
\end{aligned}
$$

where $\Delta$ is defined by (4.31). Let us put $N_{i}^{\prime} \equiv f\left(N_{i}\right)$. Then (5.11) is unchanged if we replace the $f\left(N_{i}\right)$ by the $N_{i}^{\prime}$, and maximize with respect to the $N_{i}^{\prime}$. Since $N_{i}^{\prime} \leqq \varrho^{\prime} \omega$ we have, by an argument like that leading to (4.17), that

$$
A^{0}\left(N_{i}^{\prime}, \omega\right) / \omega<a^{0}\left(N_{i}^{\prime} / \omega\right)+\varepsilon_{6}^{\prime}(\omega)
$$


where $\varepsilon_{6}^{\prime}(\omega)$ is a positive function which vanishes when $\omega \rightarrow \infty$. Thus $\varepsilon_{6}^{\prime}$ vanishes in the LP triple limit. Now (5.11) reduces to

$$
\begin{aligned}
P(\mu, \Omega, \gamma)< & \frac{1}{|\Omega|} \max _{N_{1}^{\prime} \ldots N_{M}^{\prime}}\left\{\sum_{i}\left[N_{i}^{\prime} \mu-N_{i}^{\prime} \psi_{i}^{+}-a^{0}\left(N_{i}^{\prime} / \omega\right) \omega\right]\right. \\
& \left.-\frac{1}{2} \gamma^{v} \sum_{i j} N_{i}^{\prime} N_{j}^{\prime} K_{i j}^{+}\right\}+\varepsilon_{1}^{\prime} \cdots+\varepsilon_{6}^{\prime}+\Delta .
\end{aligned}
$$

Let $\mathscr{S}_{M}$ be the class of step functions which are of the form (4.18) in $\bar{\Omega}$, and are periodic with unit cell $\bar{\Omega}$. Then from (5.13) we have

$$
\begin{aligned}
P(\mu, \Omega, \gamma) & <\sup _{n \in \mathscr{S}_{M}} F(n, \bar{\Omega})+\varepsilon_{1}^{\prime} \cdots+\varepsilon_{6}^{\prime}+\Delta \\
& \leqq \sup _{n \in \mathscr{R}} F(n, \bar{\Omega})+\varepsilon_{1}^{\prime} \cdots+\varepsilon_{6}^{\prime}+\Delta
\end{aligned}
$$

since $\mathscr{S}_{M} \subset \mathscr{R}$. Finally, taking the LP triple limit, followed by the limit $\varrho^{\prime} \rightarrow \varrho_{c}$, we have

$$
\limsup _{\gamma \rightarrow 0} p(\mu, \gamma) \leqq \lim _{|D| \rightarrow \infty} \sup _{n \in \mathscr{R}} F(n, D) .
$$

Combining this with (4.34) we deduce that $p(\mu, 0+)$ exists and is given by the statement (2.13) of Theorem 1 . The alternative statement (2.14) follows from Lemma 1, as shown in Section III.

To prove that $p(\mu, \gamma)$ tends to $p(\mu, 0+)$ uniformly on any interval, we note that for $|\mu| \leqq m$, our correction terms can be made to depend on $m$ and not on $\mu$. This completes the proof of Theorem 1 .

\section{The Free Energy}

In this section we derive results for the free energy, corresponding to those given in Theorem 1 for the pressure. The free energy of a system of $N$ particles in a cube $\Omega$ is defined by

$$
A(N, \Omega, \gamma) \equiv-\beta^{-1} \log Z(N, \Omega, \gamma)
$$

for integral $N$ and by linear interpolation [10] for non-integral $N$. The free energy density is defined by

$$
a(\varrho, \gamma) \equiv \lim _{|\Omega| \rightarrow \infty} A(\varrho|\Omega|, \Omega, \gamma) /|\Omega|
$$

which exists [9] because of conditions (2.6-12) (see the discussion just before Theorem 1). The van der Waals limit of the free energy density is defined by

$$
a(\varrho, 0+) \equiv \lim _{\gamma \rightarrow 0} a(\varrho, \gamma)
$$


The second main result of this paper is

Theorem 2. Under conditions (2.6-12), the van der Waals limit a $(\varrho, 0+)$ of the free energy density exists and is given by

or equivalently by

$$
a(\varrho, 0+)=\lim _{|D| \rightarrow \infty} \inf _{n \in \mathscr{C}_{D}(\varrho)} G(n, D)
$$

$$
a(\varrho, 0+)=\inf _{n \in \mathscr{L}(\varrho)} G(n, \infty)
$$

where $\mathscr{C}(\varrho)$ is the subclass of $\mathscr{R}$ consisting of functions $n$ which also satisfy

$$
\lim _{|D| \rightarrow \infty} \frac{1}{|D|} \int_{D} d \mathbf{y} n(\mathbf{y})=\varrho,
$$

$\mathscr{C}_{D}(\varrho)$ is the subclass of $\mathscr{C}(\varrho)$ comprising functions with unit cell $D$ and $G(n, D)$ is defined as the functional

and

$$
\begin{aligned}
\frac{1}{|D|} \int_{D} d \mathbf{y}\left\{a^{0}[n(\mathbf{y})]+\psi(\mathbf{y}) n(\mathbf{y})\right\} \\
+\frac{1}{2|D|} \int_{D} d \mathbf{y} \int_{D} d \mathbf{y}^{\prime} n(\mathbf{y}) n\left(\mathbf{y}^{\prime}\right) K\left(\mathbf{y}-\mathbf{y}^{\prime}\right)
\end{aligned}
$$

$$
G(n, \infty) \equiv \lim _{|D| \rightarrow \infty} G(n, D) .
$$

Further, the convergence of $a(\varrho, \gamma)$ to $a(\varrho, 0+)$ is uniform in $\varrho$ for $0 \leqq \varrho \leqq \varrho^{\prime}$ if $\varrho^{\prime}<\varrho_{c}$.

The limits (6.4, 6 and 8) are taken over an ascending sequence of similarly oriented cubes $D$, as in Theorem 1 . The existence of all the limits involving $|D| \rightarrow \infty$ and the infima follows from arguments like those of Section III.

Before proving Theorem 2, we note that if $\psi=0$ and the minimal function in (6.5) happens to be $n=\varrho$, then $a(\varrho, 0+)=a^{0}(\varrho)+\frac{1}{2} \alpha \varrho^{2}$, where $\alpha \equiv \int d \mathbf{s} K(\mathbf{s})$. This formula was shown by LP to hold for all $\varrho$ and all temperatures if $K$ has a non-negative Fourier transform, and for restricted ranges of $\varrho$ and of temperature if $K$ is a more general function.

Theorem can be proved from first principles by a method similar to that used for Theorem 1. Instead, we give a simpler proof using Theorem 1 together with the standard formula [15]

$$
a(\varrho, \gamma)=\max _{\mu}[\mu \varrho-p(\mu, \gamma)] .
$$

Taking the limit $\gamma \rightarrow 0$ of this gives

$$
a(\varrho, 0+)=\max _{\mu}[\mu \varrho-p(\mu, 0+)]
$$


where the interchange ${ }^{2}$ of the limit and the maximum is justified because, by Theorem $1, p(\mu, \gamma)$ tends to $p(\mu, 0+)$ uniformly in $\mu$. From the Eq. (2.14) for $p(\mu, 0+)$ we deduce that

$$
\begin{aligned}
p(\mu, 0+) & =\sup _{\varrho} \sup _{n \in \mathscr{C}(\varrho)} F(n, \mu, \infty) \\
& =\sup _{\varrho} \sup _{n \in \mathscr{C}(\varrho)} \lim _{|D| \rightarrow \infty}\left[\mu \frac{1}{|D|} \int_{D} d \mathbf{y} n(\mathbf{y})-G(n, D)\right] \\
& =\sup _{\varrho}\left[\mu \varrho-\inf _{n \in \mathscr{C}(\varrho)} G(n, \infty)\right] .
\end{aligned}
$$

Substituting (6.11) in (6.10) gives

$$
a(\varrho, 0+)=\max _{\mu} \inf _{\varrho^{\prime}}\left[\left(\varrho-\varrho^{\prime}\right) \mu+\inf _{n \in \mathscr{C}\left(\varrho^{\prime}\right)} G(n, \infty)\right] .
$$

To proceed further we need two lemmas.

Lemma 3. The function ${ }^{8}$

$$
f(\varrho) \equiv \inf _{n \in \mathscr{C}(\varrho)} G(n, \infty)
$$

is a convex function of $\varrho$

Lemma 4. If $g(\varrho)$ is convex then

$$
\max _{\mu} \min _{\varrho^{\prime}}\left[\left(\varrho-\varrho^{\prime}\right) \mu+g(\varrho)\right]=g(\varrho) .
$$

To prove Lemma 3 it is sufficient [14] to prove that $f(\varrho)$ is bounded above in some subinterval of $\left[0, \varrho_{c}\right]$, and that

$$
f\left(\frac{1}{2} \varrho_{1}+\frac{1}{2} \varrho_{2}\right) \leqq \frac{1}{2} f\left(\varrho_{1}\right)+\frac{1}{2} f\left(\varrho_{2}\right)
$$

for all $\varrho_{1}$ and $\varrho_{2}$ in $\left[0, \varrho_{c}\right)$. An obvious upper bound on $f(\varrho)$ is

$$
G(\varrho, \infty)=a^{0}(\varrho)+\frac{\varrho}{|J|} \int_{J} d \mathbf{y} \psi(\mathbf{y})+\frac{1}{2} \varrho^{2} \int d \mathbf{s} K(\mathbf{s}),
$$

which is itself bounded above in $\left[0, \varrho^{\prime}\right]$ for $0<\varrho^{\prime}<\varrho_{c}$. To prove the statement (6.15), consider a cube $D$ which is divided into $2^{v}$ identical subcubes $D_{1} \ldots D_{2^{v}}$. We choose $D$ so that its sides are parallel to and are even integral multiples of those of $J$. Then the sides of each $D_{\lambda}$ are parallel to and are integral multiples of those of $J$. The definition (6.7) implies

$$
G(n, D) \leqq \frac{1}{2^{v}} \sum_{\lambda=1}^{2^{v}} G\left(n, D_{\lambda}\right)+\Delta(D)
$$

${ }^{8}$ Note that $\inf _{n \in \mathscr{\mathscr { C }}_{D}(\varrho)} G(n, D)$ is not necessarily convex (see Eq. (7.3)). 
where

$$
\Delta(D) \equiv \frac{\varrho_{c}^{2}}{|D|} \sum_{1 \leqq \lambda<\mu \leqq 2^{v}} \int_{D_{\lambda}} d \mathbf{y} \int_{D_{\mu}} d \mathbf{y}^{\prime}\left|K\left(\mathbf{y}-\mathbf{y}^{\prime}\right)\right|
$$

The quantity $\Delta(D)$ is an upper bound on $1 /|D|$ times the interaction due to $K$ of particles that are in different subcubes and, like $\delta(D)$ in (3.6), it tends to zero as $|D| \rightarrow \infty$.

For any $\varrho$, let $\mathscr{C}_{D}(\varrho)$ and $\mathscr{C}_{D_{1}}(\varrho)$ be the subclasses of $\mathscr{C}(\varrho)$ consisting of functions with unit cells $D$ and $D_{1}$ respectively. The conditions on $D$ imply that $G\left(n, D_{\lambda}\right)=G\left(n, D_{\mu}\right)$ for all $\lambda, \mu$ and $n \in \mathscr{C}_{D_{1}}(\varrho)$. Also let $\mathscr{C}_{D}\left(\varrho_{1}, \varrho_{2}\right)$ be the subclass of $\mathscr{R}$ consisting of functions $n$ having unit cell $D$ and satisfying

$$
n_{\lambda} \equiv \frac{1}{\left|D_{1}\right|} \int_{D_{\lambda}} d \mathbf{y} n(\mathbf{y})=\left\{\begin{array}{lll}
\varrho_{1} & \text { for } & \lambda=1,2, \ldots 2^{v-1} \\
\varrho_{2} & \text { for } & \lambda=2^{v-1}+1, \ldots 2^{v}
\end{array}\right.
$$

These conditions imply

$$
\frac{1}{|D|} \int_{D} d \mathbf{y} n(\mathbf{y})=\frac{1}{2}\left(\varrho_{1}+\varrho_{2}\right)
$$

so that $\mathscr{C}_{D}\left(\varrho_{1}, \varrho_{2}\right)$ is a subclass of $\mathscr{C}_{D}\left(\frac{1}{2} \varrho_{1}+\frac{1}{2} \varrho_{2}\right)$. With (6.16) this gives

$$
\begin{aligned}
\inf _{n \in \mathscr{C}_{D}\left(\frac{1}{2} \varrho_{1}+\frac{1}{2} \varrho_{2}\right)} G(n, D) & \leqq \inf _{n \in \mathscr{C}_{D}\left(\varrho_{1}, \varrho_{2}\right)} G(n, D) \\
& \leqq \frac{1}{2^{v}} \sum_{\lambda=1}^{2^{v}} \inf _{n \in \mathscr{C}_{D_{1}}\left(n_{\lambda}\right) .} G\left(n, D_{\lambda}\right)+\Delta(D) \\
& =\frac{1}{2} \sum_{i=1,2} \inf _{n \in \mathscr{C}_{D_{1}}\left(\varrho_{2}\right)} G\left(n, D_{1}\right)+\Delta(D)
\end{aligned}
$$

Now take the limit $|D| \rightarrow \infty$ of (6.20) over an ascending sequence of cubes $D$ all related to $J$ as before. By an argument like that given in Section III to prove the equivalence of (2.13) and (2.14), we can interchange limits with infima in the resulting inequality. This gives (6.15) and completes the proof Lemma 3.

To prove Lemma 4, we write $M(\varrho)$ for the left side of (6.14). Since

$$
\min _{\varrho^{\prime}}\left[\left(\varrho-\varrho^{\prime}\right) \mu+g\left(\varrho^{\prime}\right)\right] \leqq g(\varrho)
$$


for all $\mu$, we see that $M(\varrho) \leqq g(\varrho)$. Also, since $g$ is convex, its tangent at any point $\varrho$ never lies above the curve [14]. Taking $\mu$ to be the slope of this tangent, we have $g\left(\varrho^{\prime}\right)+\left(\varrho-\varrho^{\prime}\right) \mu \geqq g(\varrho)$ for all $\varrho^{\prime}$, so that

$$
\min _{\varrho^{\prime}}\left[\left(\varrho-\varrho^{\prime}\right) \mu+g\left(\varrho^{\prime}\right)\right] \geqq g(\varrho) .
$$

It follows that $M(\varrho) \geqq g(\varrho)$, and the proof of Lemma 4 is complete $^{9}$.

Using Lemmas 3 and 4 in (6.12) we obtain (6.5). We can deduce (6.4) from (6.5) by using the analogue of Lemma 1. That the convergence of $a(\varrho, \gamma)$ is uniform in $\varrho$ can be deduced from (6.9) and the fact that the convergence of $p(\mu, \gamma)$ is uniform in $\mu$. This completes the proof of Theorem 2.

As a corollary, we note that $a(\varrho, 0+)$ is convex in $\varrho$. This follows from (6.5) and Lemma 3, or alternatively from the fact that $a(\varrho, 0+)$ is the limit of a sequence of functions $a(\varrho, \gamma)$ which are known $[10]$ to be convex.

\section{Discussion}

Our main results are Theorems 1 and 2 which prove the existence of and give expressions for the van der Waals limits $p(\mu, 0+)$ and $a(\varrho, 0+)$ of the pressure and free energy density.

These results are fairly general, being easily extended to classical lattice gases and other lattice systems that are isomorphic to these gases. A more difficult generalization would be the replacement of the hard core condition (2.7) by the condition $q(\mathbf{r})>$ constant. $|\mathbf{r}|^{-v-\varepsilon}$ for small $|\mathbf{r}|$. It may be possible to do this by using a result of Dobrushin and Minlos [16]. It may also be possible, in a more sophisticated treatment, to replace the conditions on $n$, and possibly those on $K$ and $\psi$, by the condition of Lebesgue measurability. The only place where we require Riemann integrability of $n$ is in Eq. (4.25).

The result (2.13) raises the question of the significance of the function

$$
\mathscr{P}(\mu, D) \equiv \sup _{n \in \mathscr{R}} F(n, D) .
$$

One can show by our methods that

$$
\mathscr{P}(\mu, D)=\lim _{\gamma \rightarrow 0} P\left(\mu, D_{\gamma}, \gamma\right)
$$

where $D_{\gamma}$ is a cube of volume $\gamma^{-v}|D|$, defined as the set of points $\mathbf{x}$ such that $\gamma \mathbf{x} \in D$. The limit (7.3) is thus a combined van der Waals and thermodynamic limit in which the sides of the container grow at the same rate as the range $\gamma^{-1}$ of the Kac potential. One can also define a free energy

${ }^{9}$ The same argument proves that $\sup _{\mu} \inf _{\varrho^{\prime}}\left[\left(\varrho-\varrho^{\prime}\right) \mu+f\left(\varrho^{\prime}\right)\right]=C E f(\varrho)$ for any $f$, where $C E f$ was defined in Section I. 
density $\mathscr{A}(\varrho, D)$ like (7.1) and prove the analogue of (7.2). One finds that $\mathscr{A}$ is not necessarily convex in $\varrho$ (but is probably differentiable), and is not related to $\mathscr{P}$ by an equation like (6.10); i.e. the canonical and grand canonical ensembles are not equivalent in this limit. For example, if $K(\mathbf{s})=C$, a constant, for $|\mathbf{s}| \leqq|D|^{1 / v} \sqrt{v}$ (the diagonal of $D$ ) and $\psi(\mathbf{y})=0$ for all $\mathbf{y}$, one finds that

$$
\mathscr{A}(\varrho, D)=a^{0}(\varrho)+\frac{1}{2} C|D| \varrho^{2}
$$

which is not convex at low temperatures if $C$ is negative. Possibly, $\mathscr{A}$ represents a finite system of volume $|D|$, which is, roughly speaking, small enough to be thermodynamically unstable but large enough to behave like a continuum. This may have practical application.

It is possible to rederive the results of LP [4] using our Theorems 1 and 2, and also prove that there are some functions $K$ for which $(1.2,3$ and 4) do not hold. Theorems 1 and 2 can also be used to evaluate certain correlation functions in the van der Waals limit. We hope to present this in a future paper.

Acknowledgements. We are grateful to O. E. Maly and C. Orton for helpful advice.

\section{References}

1. Kac, M.: Phys. Fluids 2, 8 (1959).

2. Baker, G. A.: Phys. Rev. 126, 2072 (1962).

3. Kac, M., G. E. Uhlenbeck, and P. Hemmer: J. Math. Phys. 4, 216 (1963).

4. Lebowitz, J. L., and O. Penrose: J. Math. Phys. 7, 98 (1966).

5. Brout, R.: Phys. Rev. 115, 824 (1959).

6. Hemmer, P. C.: J. Math. Phys. 4, 248 (1963).

7. Lebowitz, J. L., G. Stell, and S. Baer: J. Math. Phys. 6, 1282 (1965).

8. van Kampen, N. G.: Phys. Rev. 135, A 362 (1964).

9. Dobrushin, R. L.: Theory probability. USSR, 9, 566 (1964).

10. Fisher, M. E.: Arch. Rat. Mech. An. 17, 224 (1964).

11. Rudin, W.: Principles of mathematical analysis, theorem 6.11 and 10.28. Second Ed. New York: McGraw-Hill

12. Rogosinski, W.: Volume and integral, theorem 57. London: Oliver and Boyd 1952.

13. Penrose, O.: Phys. Letters 11, 224 (1964).

14. Hardy, G., J. E. Littlewood, and G. Polya: Inequalities, para 3.18 (We use 'convex' in the sense that these authors use 'continuous convex'). London: Cambridge University Press 1959.

15. Lebowitz, J. L.: Ann. Rev. Phys. Chem. 19, 389 (1968).

16. Dobrushin, R. L., and R. A. Minlos:Theory Probability, USSR. 12535 (1967), final inequality.

\section{O. Penrose}

The Open University

Walton Hall

Bletchley

Bucks, Great Britain

\section{J. Gates}

Mathematics Department

Imperial College

London, S.W. 7, Great Britain 